\title{
Issues in the Treatment of Neurological Conditions Caused by Reactivation of Varicella Zoster Virus (VZV)
}

\author{
Peter G. E. Kennedy ${ }^{1}$
}

Published online: 31 March 2016

(C) The American Society for Experimental NeuroTherapeutics, Inc. 2016

\begin{abstract}
Varicella zoster virus (VZV) is a ubiquitous neurotropic human herpesvirus. Primary infection usually causes varicella (chicken pox), after which virus becomes latent in ganglia along the entire neuraxis. Decades later, virus reactivates to produce herpes zoster (shingles), a painful dermatomally distributed vesicular eruption. Zoster may be further complicated by postherpetic neuralgia, VZV vasculopathy, myelitis, and segmental motor weakness. VZV reactivation has also been associated with giant cell arteritis. This overview discusses treatment of various conditions that often require both corticosteroids and antiviral drugs. Treatment for VZV-associated disease is often based on case reports and small studies rather than largescale clinical trials. Issues that require resolution include the optimal duration of such combined therapy, more effective treatment for postherpetic neuralgia, whether some treatments should be given orally or intravenously, the widening spectrum of zoster sine herpete, and the role of antiviral therapy in giant cell arteritis.
\end{abstract}

Keywords Varicella zoster virus · Antiviral agents · Postherpetic neuralgia $\cdot$ Corticosteroids $\cdot$ Vasculitis

Peter G. E. Kennedy

Peter.Kennedy@glasgow.ac.uk

1 Glasgow University Department of Neurology, Institute of Neurological Sciences, Queen Elizabeth University Hospital, Glasgow G51 4TF, UK

\section{Introduction}

Varicella zoster virus (VZV) is a pathogenic neurotropic human herpesvirus. Primary infection, usually in children, causes varicella ("chickenpox"), after which virus becomes latent in cranial nerve ganglia, dorsal root ganglia, and autonomic ganglia along the entire neuraxis $[1,2]$. In latently infected human ganglia, VZV is located in neurons [3, 4]. Within $9 \mathrm{~h}$ of death, only VZV gene 63 is transcribed [5]. However, the consistent detection by several groups of other VZV transcripts (VZV open reading frames 4, 21, 29, 62, 63 and 66) after this time may possibly reflect agonal attempts at VZV reactivation [6-9]. Decades later, as cell-mediated immunity to VZV declines with advancing age or when humans are immunosuppressed by disease or drug therapy, VZV reactivates to cause herpes zoster ("shingles"), a painful dermatomally distributed vesicular eruption [1-3]. VZV reactivation also produces an increasingly recognized number of acute, subacute, and chronic neurological conditions, often without rash.

There are several points to consider in the treatment of the neurological conditions caused by VZV reactivation. It is necessary to decide about oral versus intravenous (IV) therapy in different clinical scenarios, and there is a need to decide on the duration of antiviral drugs and/or corticosteroids in the various conditions. When giving corticosteroids alone for VZV reactivation it seems reasonable to also consider administering concomitant antiviral therapy to prevent potentiation of viral replication and spread. Finally, in several clinical scenarios the recommended treatment discussed is not based on controlled randomized clinical trials and, indeed, is only at the class IV level, which is that based on uncontrolled studies, case series, case reports, or expert opinion [10].

The following important VZV-associated clinical conditions due to viral reactivation will be considered, 
together with a brief coverage of both their generally accepted or potential treatment.

\section{Herpes Zoster}

Antiviral therapy has been shown to be effective in reducing the severity of symptoms associated with acute herpes zoster. All immunocompetent patients over the age of 50 years, as well as those with ophthalmic distribution, or the Ramsay Hunt syndrome (facial palsy with zoster oticus), should be treated with oral antiviral agents. There is supportive evidence that such individuals should be given oral aciclovir, famciclovir, or valaciclovir for 7-10 days within $72 \mathrm{~h}$ of the onset, which reduces the severity and duration of the acute pain [11]. Antiviral therapy does not reduce the subsequent development of postherpetic neuralgia (PHN) [11-13]. If the patient is immunocompromised and/or has neurological complications, then IV aciclovir should be administered for 7-10 days [12]. While it is possible that immunocompetent patients younger than 50 years of age may not require antiviral drugs and can be managed with analgesics [1], the author is reluctant to withhold such drugs from younger individuals. A combination of oral corticosteroids (prednisolone) with aciclovir is also effective in reducing the pain of acute herpes zoster, especially in elderly patients (provided they have no contraindication to corticosteroid therapy) but has no effect in reducing the incidence of PHN [11]. However, it should be mentioned that zoster is not always painful, especially in young people. The treatment options for zoster have been extensively reviewed elsewhere [14].

\section{PHN}

This complication is the most frequent and important of the neurological complications of herpes zoster, occurring in $>40 \%$ of patients with zoster over the age of 60 years [1]. PHN has been defined operationally as pain that persists for 3 months after the zoster rash has healed [15]. The mechanism of PHN remains unknown. While host factors such as the patient's age and immunosuppression from disease or drug therapy are known to be critical determinants for the development of PHN, there is some limited evidence that persistent VZV ganglionitis causes disease [16]. However, ganglionitis is only one possibility and other possible mechanisms for the development of PHN include damage to the nerves from the skin to the spinal cord or hyperexcitability of ganglion or spinal neurons. Established risk factors for PHN development include age $>50$ years, severe or disseminated rash, a herpes zoster viremia detectable by polymerase chain reaction (PCR), female sex, the presence of a prodrome, and severe pain at the time of presentation [11].
There is no universally accepted treatment for PHN [17]. The detailed evidence for the various clinical trials of different treatments for PHN has been described elsewhere [11, 17], and PHN and its management has been extensively reviewed elsewhere $[18,19]$. In practice, physicians generally try combinations of drugs to relieve pain. Such unfortunate patients are not infrequently referred to specialized pain clinics with the hope and expectation that pharmacological and physical treatment paradigms may be optimized. Notwithstanding such efforts, there remain some patients who are in constant severe pain that is helped very little by any therapy at all and the outlook for them is poor.

There is an evidence base for using a number of systemic drugs, though some of these can have significant side effects and, to get the optimal effect without toxicity, the dose usually needs to be titrated in individual cases. Tricyclic antidepressants such as amitriptyline and nortriptyline may alleviate PHN, and can used in combination with carbamazepine. The anticonvulsant drug gabapentin has also been used and may be effective, increasing the dose slowly to maximum levels if required, and this may also be combined with nortriptyline [12]. Pregabalin can be tried if gabapentin fails or becomes ineffective. Controlled release oxycodone, an opioid analgesic, may be used effectively to relieve PHN symptoms, as can the drug tramadol [11], but the latter should not be a first-line therapy because of its potential for abuse. Administration of corticosteroids has not been shown to be effective in PHN and, as previously indicated, giving corticosteroids during acute herpes zoster does not prevent the development of PHN. There has also been reported success with various topical therapies in PHN. For example, the application of topical $5 \%$ lidocaine patches, applied 3-4 times daily has been shown to be effective in a number of clinical trials [16], with the therapeutic response being evident within a few days. Lidocaine should not be applied to diseased skin, only to intact skin. Alternative topical agents have included aspirin and capsaicin, which is derived from chili peppers [16], but various nondrug, physical treatment modalities have been found to be ineffective in alleviating the pain of PHN. An evidence-based report of the Quality Standards Subcommittee of the American Academy of Neurology found that tricyclic antidepressants, gabapentin, pregabalin, opioids, and a lidocaine patch were found to be effective in reducing the pain of PHN [18].

The current general consensus is that PHN is not due to ongoing virus replication and that IV aciclovir does not have a beneficial effect on the development or severity of PHN [14, 19]. However, it should be mentioned in this context that in a small study IV aciclovir for 14 days followed by oral valaciclovir for 1 month produced a small improvement in the pain scale in patients with PHN [20], a finding that is consistent with the finding that elderly patients with zoster with PHN were found to have VZV DNA in their circulating 
blood mononuclear cells compared with elderly patients with zoster without PHN [21]. However, whether long-term antiviral therapy may improve the pain of PHN remains to be assessed.

It should be mentioned that there is now available an effective zoster vaccine. In the important shingles prevention study [22], using a single dose of the VOka VZV strain in $>19,000$ individuals aged $\geq 60$ years, it was found that the vaccine reduced the incidence of herpes zoster by $51.3 \%$ compared with an equivalent number of nonimmunized controls, reduced the burden of disease (pain and discomfort) by $61.1 \%$ in those who did get zoster, and reduced the incidence of PHN by $65.0 \%[12,22]$. The role of zoster vaccination in the very old ( $>85$ years) and immunosuppressed population is as yet unclear. However, some of these problems may be solved by the recent development of a new liposome-based subunit vaccine developed by GlaxoSmithKline [23].

\section{VZV Vasculopathy}

VZV vasculopathy is an important and increasingly recognized complication of VZV reactivation and is due to a productive viral infection of both large and small cerebral arteries [24]. An increasing disease spectrum of this condition now includes ischemic and hemorrhagic stroke, transient ischemic attacks, arterial dissection, temporal artery infection (see below), ischemic cranial neuropathies, cerebral venous thrombosis, peripheral thrombotic disease, and spinal cord infarction [24]. It should be appreciated, however, that several of these complications are based on individual case reports, and zoster is not a frequent cause of these complications. The clinical diagnosis may be difficult, especially when not temporally associated with the typical zosteriform rash, but the condition is usually characterized by magnetic resonance imaging and computed tomography features of cerebral ischemia or hemorrhage, which may affect both gray and white matter, evidence of narrowing and/or beading of cerebral arteries seen on angiography, and a pleocytosis with mononuclear cells detected in the cerebrospinal fluid (CSF) [25]. Typically, a patient with VZV vasculopathy may present with progressive cognitive decline and confusion, seizures, and focal neurological deficits [1], but the clinical presentation is protean [24]. Infected arteries contain Cowdry A inclusion bodies, multinucleated giant cells, herpes virions, and both VZV DNA and antigen [26]. Both PCR to detect VZV DNA in the CSF and detection of anti-VZV IgG antibody in the CSF have been used successfully to diagnose VZV vasculopathy, but studies have shown, perhaps somewhat surprisingly, that this latter serological method is a more sensitive diagnostic marker of this condition than PCR analysis [25]. It is important to measure the ratio of
anti-VZV antibody/total IgG in the CSF and blood, and not just the total anti-VZV antibody.

Owing to the relatively small number of cases studied - there have not been large clinical trials - the current recommended treatment of VZV vasculopathy in immunocompetent individuals is 14 days of IV aciclovir and 5-7 days of oral corticosteroids (prednisone/prednisolone), though immunocompromised individuals or those relapsing with recurrent VZV vasculopathy may need a longer course of aciclovir and corticosteroids [24]. Consideration should also be given for long-term valaciclovir in the presence of recurrent VZV vasculopathy due to immunosuppressive or immunomodulatory drugs.

\section{VZV Myelitis and Focal Motor Weakness}

VZV is well recognized to produce an acute or chronic myelopathy that is rarely recurrent [1]. Presenting usually within 1-2 weeks of the typical zosterifrom rash, the presentation is a typical myelitis with acute bilateral leg weakness, sphincter disturbance, and a mild CSF pleocytosis and normal or moderately raised protein. The magnetic resonance imaging of the affected spinal cord is critical in diagnosis, showing T2 hyperintensities and focal swelling [1]. Diagnosis can be confirmed by PCR detection of VZV DNA in CSF. Some patients with herpes zoster may also develop a segmental motor weakness affecting, for example, a limb, the diaphragm, and intercostal or sphincter muscles, with a topographic dissociation between the zosteriform rash and focal weakness occurring in about $10 \%$ of cases [27]. The prognosis is generally thought to be favorable, with a complete recovery likely in up to $75 \%$ of cases [27]. While there is no solid evidence base for particular treatment regimes in VZV myelitis and motor weakness, the author would treat such patients with IV aciclovir for 14 days with 5-7 days of corticosteroids (oral prednisolone).

\section{Zoster Sine Herpete}

Zoster sine herpete ( $\mathrm{ZSH})$ occurs when the typical dermatomal distribution of pain occurs in the absence of an antecedent zosteriform rash. This entity was recognized as a distinct VZV-associated condition when Gilden et al. [28] reported 2 men in their 60s suffering from chronic thoracic distribution radicular pain in whom PCR-amplifiable VZV DNA was detected in the CSF 5 and 8 months, respectively, after the onset of pain, and who responded well to IV aciclovir treatment [28]. ZSH has become an increasingly recognized complication of VZV reactivation, with its range of clinical presentations widening significantly over the last few years. A diagnosis of ZSH should always be considered in the relevant 
clinical context, particularly where there is a history of persistent radicular pain. It is now clear that VZV reactivation without rash can cause many, if not all, of the various neurological disorders known to be associated with herpes zoster, including VZV vasculopathy, where $>40 \%$ of cases may occur without any antecedent rash $[25,29]$. ZSH should at least be considered in an individual who presents with acute, subacute, or chronic brain or spinal cord disease of unknown cause, especially if accompanied by a CSF pleocytosis. Whether or not the specific VZV tests are carried out will depend on the individual case presentation and the clinical assessment. The diagnosis is established by carrying out PCR for VZV DNA, as well as a search for anti-VZV IgG in CSF. The patient's blood mononuclear cells should also be examined for VZV DNA by PCR [29]. Where ZSH is diagnosed unambiguously the recommended treatment is, again, with antiviral drugs and probably also corticosteroids, but no formal clinical trial data are available. A 14-day course of IV aciclovir and 5-7 days of oral prednisolone would seem appropriate in such cases.

\section{Giant Cell Arteritis}

Giant cell arteritis (GCA) is one of the most important neurological diseases because of its potential to cause sudden and irreversible blindness and other central nervous system complications, making prompt diagnosis and immediate initiation of corticosteroid therapy a medical emergency [30]. GCA is characterized pathologically by inflammation in and damage to the vessel wall of the temporal arteries (TAs) [31, 32]. The condition may be strongly suspected on clinical grounds, with symptoms including headache, scalp tenderness, weight loss, malaise, and raised erythrocyte sedimentation rate, and the diagnosis is confirmed by a TA biopsy showing the typical inflammatory histopathological features. However, some patients who present with a typical history suggesting GCA prove to have negative biopsies and this can be very problematic, especially as a decision then has to be made about the treatment and duration of corticosteroid therapy in such biopsy-negative patients.

In this context, recent studies of GCA by Gilden et al. [32] have led to a fundamental re-evaluation of the treatment of GCA. They found that $74 \%$ of TAs in GCA-positive cases contained VZV antigen, mostly in skip (multiple noncontiguous) areas, whereas only $8 \%$ of normal TAs did so. Typical inflammatory histopathology was detected in $89 \%$ of GCA-positive TAs but not in any of the 18 adjacent tissue sections from normal TAs [32]. A very recent follow-up study by these authors found that VZV antigen was present in $64 \%$ of GCA-negative TAs compared with $22 \%$ of normal TAs [33]. They concluded that in patients with clinically suspected GCA, the prevalence of VZV in their TAs is similar, irrespective of whether the TA biopsy results are negative or positive histopathologically. While these exciting data need to be confirmed, and a cause and effect relation has yet to be proven unequivocally, they clearly indicate the potential value of treating both GCA-positive and GCA-negative patients with corticosteroids and antiviral agents. Whether oral antiviral agents and corticosteroids will be as effective as IV aciclovir and corticosteroids is uncertain. Given the VZV burden in infected TAs, oral antivirals should probably be continued after corticosteroids have been discontinued.

Required Author Forms Disclosure forms provided by the authors are available with the online version of this article.

\section{References}

1. Gilden DH, Kleinschmidt-DeMasters BK, LaGuardia JJ, Mahalingam R, Cohrs RJ. Neurologic complications of the reactivation of varicella-zoster virus. N Engl J Med 2000; 342: 635-645.

2. Mitchell BM, Bloom DC, Cohrs RJ, Gilden DH, Kennedy PGE. Herpes simplex virus-1 and varicella-zoster virus latency in human ganglia. J Neurovirol 2003;9:194-204.

3. Kennedy PGE, Cohrs RJ Varicella-zoster virus human ganglionic latency — a current summary. J Neurovirol 2010;16:411-418.

4. Kennedy PGE, Grinfeld E, Gow JW. Latent varicella-zoster virus is located predominantly in neurons in human trigeminal ganglia. Proc Natl Acad Sci U S A 1998;95:4658-4662.

5. Ouwendijk WJ, Choe A, Nagel MA, et al. Restricted varicella-zoster virus transcription in human trigeminal ganglia obtained soon after death. J Virol 2012;86: 10203-10206.

6. Kennedy PGE, Grinfeld E, Bell JE. Varicella-zoster virus gene expression in latently infected human trigeminal ganglia. J Virol 2000;74:11893-11898.

7. Kennedy PGE, Rovnak J, Badani H, Cohrs RJ. A comparison of HSV-1 and VZV latency and reactivation. J Gen Virol 2015; 96: 1581-602.

8. Cohrs RJ, Randall J, Smith J, et al. Analysis of individual human trigeminal ganglia for latent herpes simplex virus type 1 and varicella-zoster virus nucleic acids using real-time PCR. J Virol 2000;74: 11464-11471.

9. Nagel MA, Choe A, Traktinskiy I, Cordery-Cotter R, Gilden D, Cohrs RJ. Varicella-zoster virus transcriptome in latently infected human ganglia. J Virol 2011; 85:2276-2287.

10. Steiner I, Budka,H, Chaudhuri A, et al. Viral encephalitis: guidelines for diagnosis and management. Eur J Neurol 2005; 12 : 331-343.

11. Wareham DW, Breuer J. Herpes zoster. BMJ 2007; 334:1211-1215.

12. Gershon A, Breuer J, Cohen J, et al. Varicella zoster virus infection. Nat Rev Primers 2015; 1: 15016.

13. Acosta EP, Balfour HH. Acyclovir for treatment of postherpetic neuralgia: efficacy and pharmacokinetics. Antimicrob Agents Chemother 2001; 45: 2771-2774.

14. Dworkin RH, Johnson RW, Breuer J, et al. Recommendations for the management of herpes zoster. Clin Infect Dis 2008;47:303-327.

15. Kennedy PGE, Montague P, Scott F, et al .Varicella-zoster viruses associated with post-herpetic neuralgia induce sodium current density increases in the ND7-23 Nav-1.8 neuroblastoma cell line. PLoS ONE 2013; 8 :e51570. 
16. Gilden DH, Cohrs RJ, Mahalingam R.VZV vasculopathy and postherpetic neuralgia: progress and perspective on antiviral therapy. Neurology 2005; 64: 21-25.

17. Argoff CE, Katz N, Backonja M. Treatment of postherpetic neuralgia: a review of therapeutic options. J Pain Symptom Manage 2004; 28:396-411.

18. Dubinsky RM, Kabbani H, El-Chami Z, Boutwell C, Ali H. Practice parameter: treatment of postherpetic neuralgia. An evidence-based report of the Quality Standards Subcommittee of the American Academy of Neurology. Neurology 2004; 63:959-965

19. Johnson RW, Rice ASC. Postherpetic neuralgia. N Engl J Med 2014; 371:1526-1533.

20. Quan D, Hammack BN, Kittelson J, Gilden DH. Improvement of postherpetic neuralgia after treatment with intravenous acyclovir followed by oral valacyclovir Arch Neurol 2006; 63:940-942.

21. Mahalingam R, Wellish M, Brucklier J, Gilden DH Persistence of varicella-zoster virus DNA in elderly patients with postherpetic neuralgia. J Neurovirol 1995; 1:130-133.

22. Oxman MN, Levin MJ, Johnson GR, et al A vaccine to prevent herpes zoster and postherpetic neuralgia in older adults. N Engl J Med 2005; 352:2271-2284.

23. Lal H, Cunningham AL, Godeaux O, et al. Efficacy of an adjuvanted herpes zoster subunit vaccine in older adults. N Engl $\mathrm{J}$ Med 2015;372:2087-2096

24. Nagel MA, Gilden D. Update on varicella zoster virus vasculopathy. Curr Infect Dis Rep 2014; 16:407.
25. Nagel MA, Forghani B, Mahalingam R, et al The value of detecting anti-VZV IgG antibody in CSF to diagnose VZV vasculopathy. Neurology 2007; 68:1069-1073.

26. Gilden DH, Kleinschmidt-DeMasters BK, Wellish M, Hedley-Whyte ET, Rentier B, Mahalingam R. Varicella zoster virus, a cause of waxing and waning vasculitis: the New England Journal of Medicine case 5-1995 revisited. Neurology 1996 ;47: 1441-1446.

27. Kennedy PGE. Neurological complications of varicella-zoster virus infections. In Kennedy PGE, Johnson RT (eds) Infections of the Nervous System. Butterworths, 1987; 177-208.

28. Gilden DH, Wright RR, Schneck SA, Gwaltney JM Jr, Mahalingam R. Zoster sine herpete, a clinical variant Ann Neurol 1994; 35:530-533.

29. Kennedy PGE. Zoster sine herpete: it would be rash to ignore it. Neurology 2011; 76:416-417.

30. Smith JH, Swanson JW. Giant cell arteritis. Headache 2014;54: 1273-1289.

31. Kennedy PGE, Lipton HL Varicella-zoster virus claims yet another painful scalp: giant cell arteritis. Neurology 2015; 84: 1918-1919.

32. Gilden D, White T, Khmeleva N, et al Prevalence and distribution of VZV in temporal arteries of patients with giant cell arteritis. Neurology 2015; 84:1948-1955.

33. Nagel MA, White T, Khmeleva N, et al. Analysis of varicella-zoster virus in temporal arteries biopsy positive and negative for giant cell arteritis. JAMA Neurol 2015; 72:1281-1287. 